\title{
Anti-Tumor Necrosis Factor Antibody Augments Edema Formation in Caerulein-Induced Acute Pancreatitis ${ }^{1,2}$
}

\author{
K. S. Guice, M.D., K. T. Oldham, M.D.,* D. G. Remick, M.D., $†$ S. L. Kunkel, Ph.D. $\dagger$ ann P. A. Warn, M.D. $\dagger$ \\ *Sections of General Surgery and Pediatric Surgery, $\dagger$ Departments of Surgery and Pathology, \\ the University of Michigan School of Medicine, Ann Arbor, Michigan 48109 \\ Submitted for publication November 20, 1991
}

\begin{abstract}
The pathogenesis of acute pancreatitis is incompletely defined, but the outcome is determined in part by an acute inflammatory process. Pancreatitis-associated inflammation appears to play a role in the local retroperitoneal injury as well as in the associated dysfunction of remote organs such as the lung. Tumor necrosis factor (TNF) appears to be a proximal mediator of the inflammatory response. In this study, anti-TNF antibody was administered to rats with caerulein-induced pancreatitis to determine if the observed increases in pancreatic and pulmonary microvascular permeability were related to plasma TNF activity. In contrast to the expected findings, blockade of TNF activity was found to increase the amount of edema formation in both the pulmonary and pancreatic microvascular beds. The mechanism is not known; however, blockade of TNF-induced down regulation of phagocytic cell activity, ablation of TNF-dependent feedback inhibition of other cytokines, failure of induction of endogenous antioxidant systems, or inactivation of the TNF control of microvascular tone are all possible explanations. This is potentially an important observation as clinical strategies are now being developed to modify the infiammatory response in ways presumed advantageous to an injured host. (c) 1991 Academic Press, Inc.
\end{abstract}

\section{INTRODUCTION}

Acute pancreatitis is an enigmatic clinical process which is typically associated with local edema formation and acute inflammation. Some patients develop progressive pancreatic necrosis and multiorgan system failure. The factors which regulate these processes are unknown. An important mediator of the inflammatory process in other experimental injury models is tumor necrosis factor $\alpha$ (TNF) [1]. TNF is derived from activated macrophages and appears to have important effects

\footnotetext{
${ }^{1}$ Presented at the Annual Meeting of the Association for Academic Surgery, Houston, TX, November 14-17, 1990.

${ }^{2}$ Supported in part by NIH Grants HL38141 and GM41745.
}

upon the microvasculature. In particular, TNF appears to increase microvascular permeability by virtue of several effects upon the endothelial cell. TNF-mediated endothelial cell permeability increases have been related in vitro to the induction of membrane adhesion molecules which provide a means for neutrophil adherence and subsequent injury to occur. In addition, the secretion of interleukins 1 (IL-1) and 6 (IL-6), increased synthesis of prostaglandin $I_{2}$ (prostacyclin), and direct morphological alterations of endothelial cells all result from exposure to TNF [2].

Caerulein-induced acute pancreatitis in rats is associated with endothelial cell injury and edema formation in both the pancreatic and pulmonary microvasculature [3-5]. This appears to be neutrophil related. The purpose of this study was to evaluate whether TNF is a mediator of edema formation in this model of caeruleininduced acute pancreatitis. The data suggest that TNF is involved in the process of both pancreatic and lung edema formation, although not simply as a noxious mediator.

\section{MATERIALS AND METHODS}

\section{Animal Model}

All experimental protocols received prior approval from the University of Michigan Committee for the Use and Care of Animals. Male Sprague-Dawley rats (150$200 \mathrm{~g}$, Charles River, Portage, MI) were anesthetized using ketamine hydrochloride $(100 \mathrm{mg} / \mathrm{kg}$ body weight) given subcutaneously. Silastic central venous catheters were placed by cutdown into the jugular vein and tunneled to exit the dorsal cervical region of each rat. The rats were allowed to recover overnight. The following day, rats were assigned to receive either caerulein $(5 \mu \mathrm{g} /$ $\mathrm{kg} / \mathrm{hr}$, Sigma Chemical Co., St. Louis, MO) or sterile normal saline solutions using a continuous intravenous infusion of $2 \mathrm{ml} / \mathrm{hr}$ (Pump 22, Harvard Co., South $\mathrm{Na}$ tick, MA). This caerulein dose is known to induce acute edematous pancreatitis in rats [6]. Rats were infused for $0.5,1,3,6$, or $12 \mathrm{hr}$. After completion of infusion, the rats 
were sacrificed and venous blood was obtained for TNF assay.

In the second portion of the experiment, rats were prepared with central venous catheters as described above. Prior to infusion, rats received an intravenous injection ( $1 \mathrm{cc}$ ) of either rabbit-derived anti-TNF antiserum (anti-TNF) or normal rabbit serum (NRS) (serum and antiserum diluted 1:1 with sterile normal saline prior to injection). Three hours prior to sacrifice, each animal received a $1 \mathrm{ml}$ intravenous bolus of $\left[{ }^{125} \mathrm{I}\right]-$ bovine serum albumin (BSA, $800,000 \mathrm{cpm} / \mathrm{ml}$ ). After 3 or $12 \mathrm{hr}$ of caerulein infusion, ketamine hydrochloride $(100 \mathrm{mg} / \mathrm{kg}$ body weight) was administered subcutaneously and laparotomy was performed. One milliliter of blood was aspirated from the inferior vena cava, the heart and lungs were quickly excised, the pancreas was removed, and the animals were exsanguinated. Twenty milliliters of sterile saline was infused into the right ventricle to wash residual blood and ${ }^{125}$ I-BSA from the pulmonary vascular bed. Done quickly, a spontaneous heart beat persisted, the pulmonary parenchyma blanched, and the effluent became asanguinous. The blood sample, pancreas, and lungs were then counted in a gamma scintillation counter. Results are expressed as a permeability index (number of ${ }^{125} \mathrm{I}$ counts in tissue divided by the number of ${ }^{125} I$ counts in the blood) for each rat. This ratio reflects albumin sequestration in tissue and is thus correlated with microvascular permeability $[3,4]$.

\section{Assay for Plasma TNF Activity}

TNF activity was determined using the WEHI 164, subclone 13 cell line. This bioassay, which depends upon the lytic effect of TNF on this mouse fibrosarcoma line is the most sensitive available assay (capable of detecting approximately $1 \mathrm{pg} / \mathrm{ml}$ of TNF) [7-9]. Briefly, plasma samples were serially diluted and placed in 96 well microtiter plates (Costar, Cambridge, MA). The WEHI cells were resuspended at $5 \times 10^{5}$ cells $/ \mathrm{ml}$ in RPMI 1640, $10 \%$ fetal calf serum, $1 \mathrm{~m} M_{\mathrm{L}}$-glutamine, 0.5 $\mathrm{ml}$ actinomycin $\mathrm{D}$ (Calbiochem, La Jolla, CA), and added to the diluted test samples $(100 \mu \mathrm{l} /$ well $)$. The plates were incubated at $37^{\circ} \mathrm{C}$ and $5 \% \mathrm{CO}_{2}$ for $20 \mathrm{hr}$. Following incubation, $20 \mathrm{ml}$ of MTT-tetrazolium ( $5 \mathrm{mg} / \mathrm{ml}$, Sigma) in phosphate-buffered saline was added to each well and the plates were reincubated for 4 additional $\mathrm{hr}$. After the second incubation, $100 \mathrm{ml}$ of $0.04 \mathrm{~N} \mathrm{HCl}$ /isopropanol was added to each well to dissolve the purple formazine crystals. Plates were stored overnight in the dark and then read at $550 \mathrm{~nm}$ on a Bio-Kinetics Reader (Bio-Tek Instruments, Inc., Winoski, VT). Readings were compared to a standard curve generated using human recombinant TNF (Cetus Corp., Emeryville, CA) and data are expressed as units $/ \mathrm{ml}$. Confirmation of the cytotoxicity of plasma TNF in the positive samples was achieved by neutralization with a polyclonal rabbit antimurine TNF antiserum (anti-TNF antibody) which cross-reacts with rat TNF.
A polyclonal rabbit antimurine-TNF antiserum (antiTNF) was used to assess the effects of TNF blockade on microvascular permeability in both the pulmonary and pancreatic vascular beds. The antibody neutralizes both recombinant and natural TNF and cross-reacts with rat TNF [9]. At a dilution of 1:10,000 the antibody will completely neutralize 10 units of rat TNF. (Twenty two units equal $1 \mathrm{ng}$ of TNF).

\section{Endotoxin Assay}

The absence of endotoxin in this experimental model was confirmed using a commercial limulus amebocyte lysate assay (Whittaker MA Bioproducts, QCC-1000 TM, Walkersville, MD). Plasma samples for endotoxin measurement were taken simultaneously with samples for TNF measurement. Briefly, $1 \mathrm{ml}$ of blood from the inferior vena cava was drawn into a heparinized syringe and lysate inhibitory factors were removed by pretreatment with $2.53 \% \mathrm{HClO}_{4}$ and $0.2 \mathrm{~N} \mathrm{NaOH}$. The assay depends upon bacterial endotoxin to activate a proenzyme in the limulus amoebocyte lysate which catalyzes the cleavage of $p$-nitroanaline (pNA) from the colorless substrate. The pNA is assayed spectrophotometrically at $405 \mathrm{~nm}$ and provides a quantitative analysis of endotoxin content. Samples were compared to the absorption of serially diluted reference endotoxin standards (Escherichia coli endotoxin 0111; B4, Difco, Detroit, MI).

\section{Statistical Analysis}

Data are expressed as means \pm standard error of the mean. A one-way analysis of variance (ANOVA) was used with post hoc comparison using Fisher's protected least squares difference to analyze the data. Significance was assigned when $P<0.05$.

\section{RESULTS}

Plasma TNF was measured in systemic blood to determine whether significant elevations occurred in this model of acute pancreatitis. The data in Fig. 1 illustrate a stepwise increase in systemic plasma TNF levels during the induction of acute pancreatitis. Only small amounts of TNF $(0.13 \pm 0.09$ units $/ \mathrm{ml})$ are detectable in the plasma of rats prior to caerulein infusion (Time 0 ). Plasma levels begin to rise by $60 \mathrm{~min}$ of caerulein infusion $(0.32 \pm 0.09$ units $/ \mathrm{ml})$ and by $180 \mathrm{~min}$ of caerulein infusion, there is a fourfold increase $(P<0.05)$ in TNF levels compared to controls. Previous data demonstrate well-established acute edematous pancreatitis at $3 \mathrm{hr}$ with more severe disease including focal pancreatic necrosis after $12 \mathrm{hr}[3,5,6]$. Plasma TNF levels after $6 \mathrm{hr}$ returned to near baseline levels; however, a biphasic response is apparent when the infusions continued to 12 $\mathrm{hr}$. In separate experiments using infusions of $12 \mathrm{hr}$ duration, plasma TNF levels were increased fourfold in animals with caerulein-induced acute pancreatitis com- 


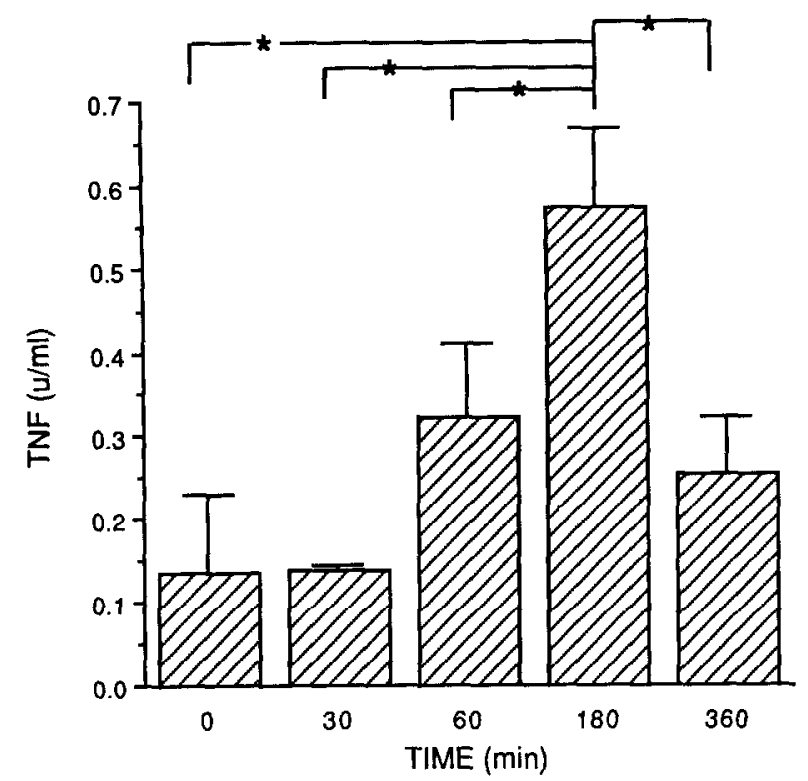

FIG. 1. Plasma TNF levels following the induction of caeruleininduced acute pancreatitis. Prior to the onset of acute pancreatitis (Time 0) there is little TNF present $(0.31 \pm .09$ units $/ \mathrm{ml})$. After 180 min there is a fourfold increase in the amount of TNF present $(P<$ $0.05)$.

pared to saline infused controls $(11.08 \pm 6.06$ units $/ \mathrm{ml}$ versus $3.07 \pm 1.28$ units $/ \mathrm{ml}$, respectively, $P<0.05$ ). Because the WEHI 164 assay is a bioassay, absolute TNF values are variable, reflecting day to day changes in the cytotoxicity threshold of the fibrosarcoma target cells. Therefore, quantitative comparisons require concurrent controls (as provided), but are not possible between experiments. For this reason, these data and the data in Fig. 1 can be compared only qualitatively. In this regard, a consistent biphasic pattern is observed with an early TNF peak (1-3 hr), returning to near normal $(6 \mathrm{hr})$ and a later TNF peak (12 hr) among experimental animals with caerulein-induced acute pancreatitis.

Plasma was assayed for endotoxin to ensure that these TNF elevations were not secondary to endotoxin, a potent stimulus of TNF release. No endotoxin was detected in any control or experimental samples (data not shown).

Data showing pancreatic edema formation are shown in Figs. 2 and 3. After $3 \mathrm{hr}$ (Fig. 2) or $12 \mathrm{hr}$ (Fig. 3) of caerulein infusion, there was a significant increase in pancreatic edema formation in all animals with caerulein-induced pancreatitis compared to normal saline-infused control animals. The experimental group receiving caerulein and anti-TNF antibody had an additional significant increase in pancreatic edema formation compared to the groups receiving caerulein alone or caerulein plus normal rabbit serum.

Lung edema formation is shown in Fig. 4 (3-hr caerulein infusion) and Fig. 5 (12-hr caerulein infusion) as the edema index. Lung edema formation in the groups re-

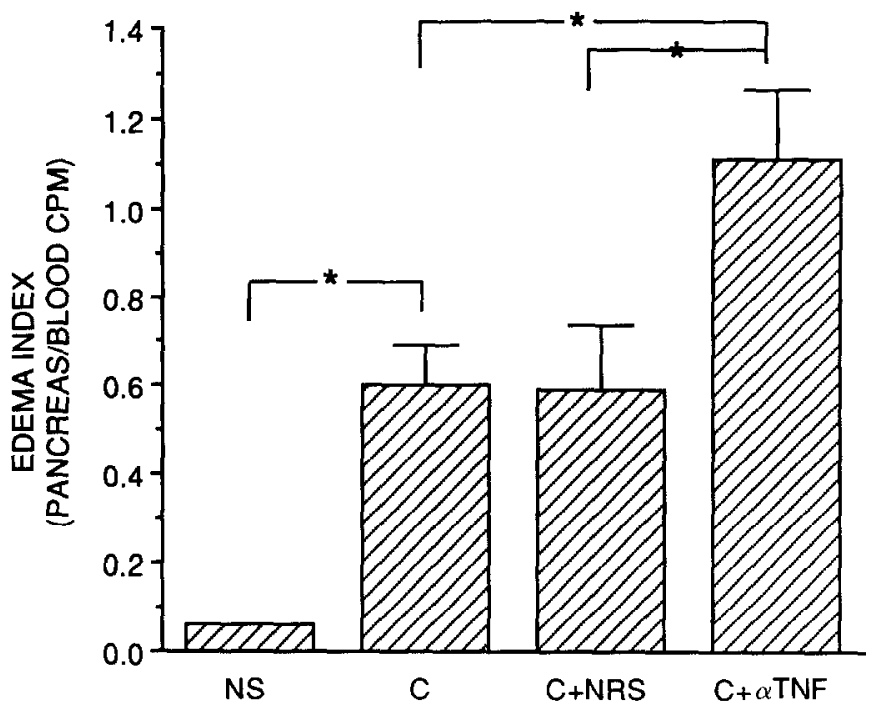

FIG. 2. Pancreas permeability after 3 hours of infusion is represented by the edema index (the number of counts in tissue/the number of counts in blood). A significant increase in edema occurs in the caerulein (C) group, the caerulein + normal rabbit serum (C + NRS) group, and the caerulein + anti-TNF $(\mathrm{C}+\alpha \mathrm{TNF})$ group compared to the normal saline group. In addition there is a significant increase in the amount of edema present in the $\mathrm{C}+\alpha \mathrm{TNF}$ group compared to the $\mathrm{C}$ or the $\mathrm{C}+\mathrm{NRS}$ group $\left({ }^{*} P<0.05\right)$.

ceiving caerulein and anti-TNF antibody was significantly $(P<0.05)$ increased over all the other groups at both the 3 - and 12-hr time points.

\section{DISCUSSION}

The roles of cytokines in general and, TNF in particular, are not clearly defined in the inflammatory process

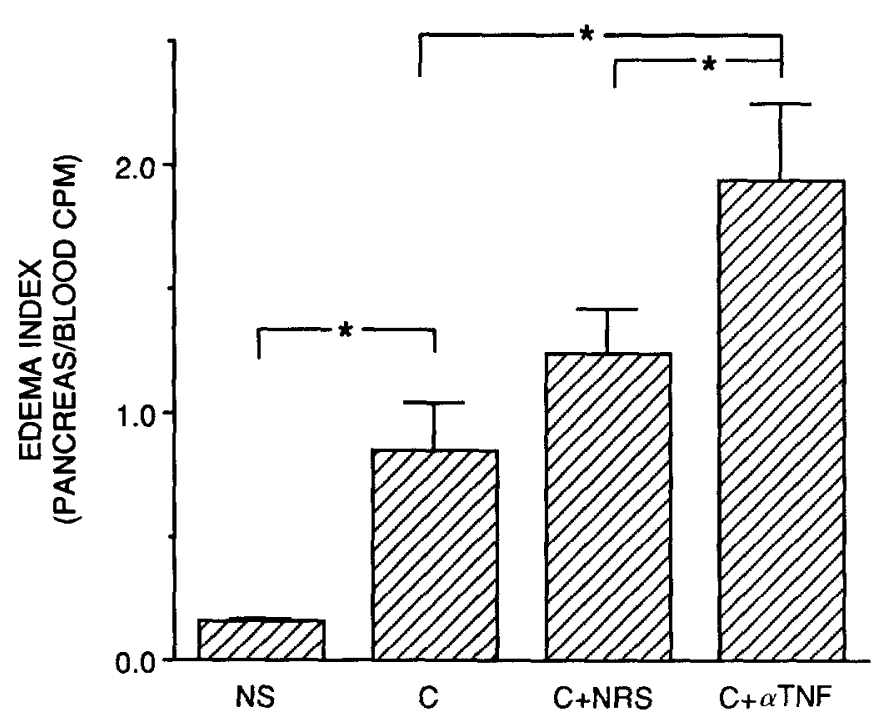

FIG. 3. After $12 \mathrm{hr}$ of caerulein infusion, the increase in pancreatic edema formation seen at $3 \mathrm{hr}$ of infusion remains. There is a significant increase in the amount of edema present in the $\mathrm{C}+\alpha \mathrm{TNF}$ group compared to all other groups $\left({ }^{*} P<0.05\right)$. 


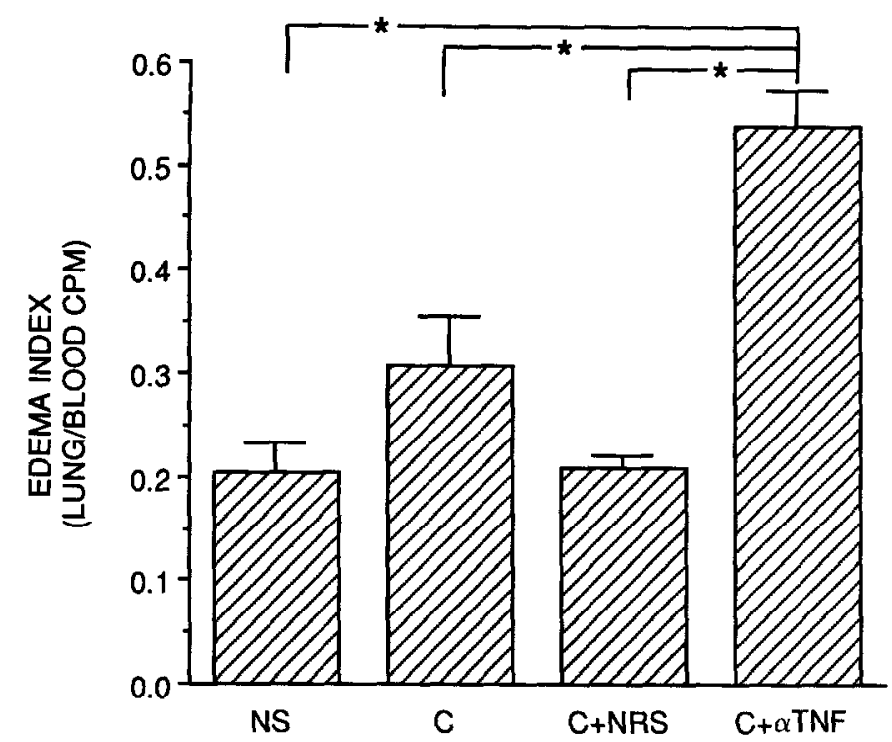

FIG. 4. Lung edema in the group receiving $\mathrm{C}+\alpha \mathrm{TNF}$ is significantly increased over the $\mathrm{C}$ and the $\mathrm{C}+\mathrm{NRS}$ groups after $3 \mathrm{hr}$ of caerulein infusion.

which accompanies caerulein-induced acute pancreatitis. TNF-mediated effects on neutrophil and endothelial cell function are described in other experimental systems and are usually associated with an increase in microvascular permeability. These effects include the induction of adhesive surface molecules on endothelial cell membranes, a neutrophil chemotactic response, neutrophil activation, cytokine-cytokine interactions, and undoubtedly others not yet elucidated [2]. In addition, TNF-induced oxidant generation by phagocytic cells may play a role in this model, as it is known that pancreatic injury is in part oxygen radical mediated [3]. The physiological response of endothelial cell exposure to TNF is an increase in microvascular permeability.

Given these published data, the initial findings in this study of parallel increases in plasma TNF levels and the degree of edema formation in lung and pancreas were expected. However, the development of significant additional edema formation after anti-TNF antibody pretreatment was not anticipated. The mechanisms responsible for this apparent paradox are not known nor is the explanation for the early and late TNF peaks. With regard to the latter, presumably clearance of the early TNF peak is followed by a second proinflammatory stimulus.

Gordon and Sheppard demonstrated that small doses of endotoxin or recombinant human TNF prevented airway edema formation in guinea pigs exposed to $80 \%$ toluene 2,4-diisocyanate, $20 \%$ toluene 2,6-diisocyanate [10]. In this report, airway edema formation was not prevented when anti-TNF antibody or heat-inactivated TNF were given. The authors speculated that TNF may inhibit airway edema formation by nonspecifically altering or inhibiting the ability of neutrophils or endothelial cells to respond to a subsequent inflammatory challenge.

Mier and colleagues found that TNF pretreatment of cultured human endothelial cells derived from the saphenous vein prevented diffusion of labeled BSA when the cells were subsequently treated with lymphokine-activated killer cells [11]. Their results suggest that TNF may protect endothelial cells from interleukin 2-induced capillary leak.

The data from the current study suggest that TNF is indeed involved in the process of edema formation and endothelial cell injury in this model. Edema formation is significantly enhanced in both lungs and pancreatic tissue in caerulein-induced pancreatitis when TNF activity is blocked with specific antibody. This suggests that some endogenous TNF-mediated inhibitory process is altered when TNF activity is blocked. Possible explanations include (1) loss of TNF-induced down regulation of inflammatory effector cells, (2) blockade of TNF-mediated feedback inhibition, perhaps involving other cytokines such as IL-2, (3) failure of endogenous antioxidant induction, or (4) alterations in the regulation of TNF-mediated microvascular tone.

In summary, our findings suggest that plasma TNF may participate in a sequence of regulatory events that determine the degree of lung and pancreatic microvascular permeability increase associated with this model of caerulein-induced acute pancreatitis. The importance of the observation is that evolving therapeutic strategies which involve alteration or modification of the inflam-

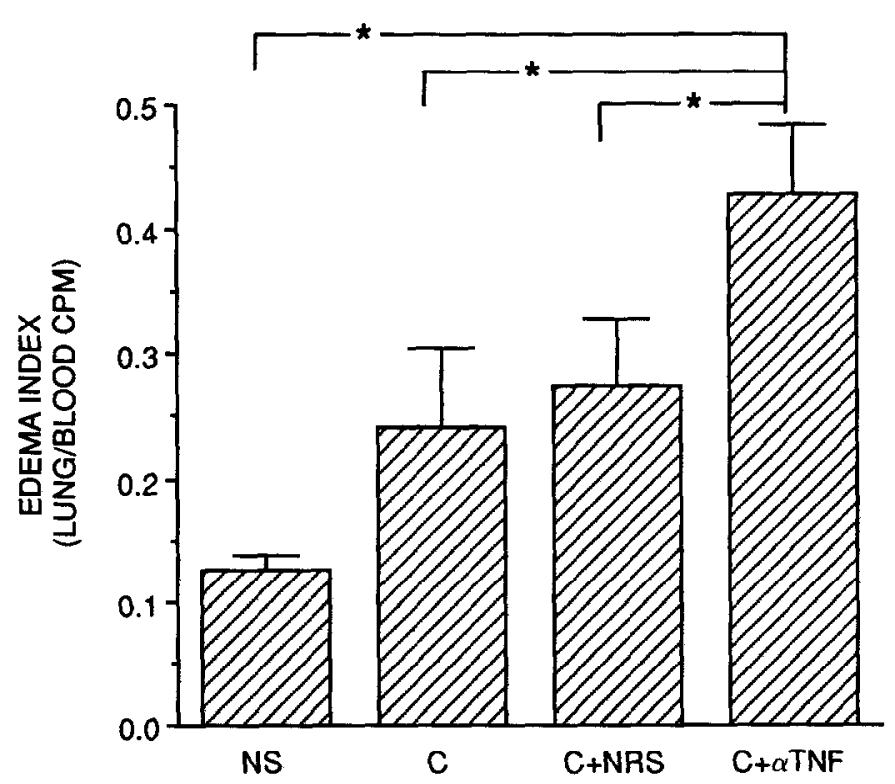

FIG. 5. After $12 \mathrm{hr}$ of caerulein infusion, a significant elevation in lung edema persists in the $\mathrm{C}+\alpha \mathrm{TNF}$ group compared to the $\mathrm{C}$ or the $\mathrm{C}+$ NRS groups $\left({ }^{*} P<0.05\right)$. 
matory process may have important additional effects which may be undesirable.

\section{REFERENCES}

1. Remick, D. G., Kunkel, R. G., Larrick, J. W., and Kunkel, S. L. Acute in vivo effects of human recombinant tumor necrosis factor. Lab Invest 56: 583, 1987.

2. Pober, J. S. TNF as an activator of vascualr endothelium. Ann. Inst. Pasteur Immunol. 139(3): 317, 1988.

3. Guice, K. S., Oldham, K. T., Johnson, K. J., and Ward, P. A. Mechanisms of pancreatic capillary endothelial injury in acute pancreatitis. Surg. Forum 38: 144, 1987.

4. Guice, K. S., Oldham, K. T., Caty, M. G., Johnson, K. J., and Ward, P. A. Neutrophil-dependent, oxygen-radical mediated lung injury associated with acute pancreatitis. Ann. Surg. 210: 740, 1989.

5. Guice, K. S., Oldham, K. T., Johnson, K. J., Kunkel, R. G., Morganroth, M. L., and Ward, P. A. Pancreatitis-induced acute lung injury: An ARDS model. Ann. Surg. 208: 71, 1988.
6. Lampel, M., and Kern, H. F. Acute interstitial pancreatitis in the rat induced by excessive doses of a pancreatic secretagogue. $\mathrm{Vir}$ chows Arch. 373: 97, 1977. [Abstract]

7. Espevik, T., and Nissen-Meyer, J. A highly sensitive cell line, WEHI 164 clone 13, for measuring cytotoxic factor/tumor necrosis factor from human monocytes. J. Immunol. Methods 95: 99 , 1986.

8. Remick, D. G., Strieter, R. M., Eskandari, M. K., Nguyen, D. T., Genord, M. A., Raiford, C. L., and Kunkel, S. L. Role of tumor necrosis factor $\alpha$ in lipopolysaccharide induced pathologic alterations. Am. J. Pathol. 136(1): 1990.

9. Chensue, S. W., Remick, D. G., Shmyr-Forsch, C., Beals, T. F., and Kunkel, S. L. Immunohistochemical demonstration of cytoplasmic and membrane-associated tumor necrosis factor in murine macrophages. Am. J. Pathol. 133 (3): 564, 1988.

10. Gordon, T., and Sheppard, D. Tumor necrosis factor inhibits a polymorphonuclear leukocyte-dependent airway edema in guinea pigs. J. Appl. Physiol. 64(4): 1688, 1988.

11. Mier, J. W., Brandon, E: P., Libby, P., Janicka, M. W., and Aronson, F. R. Activated endothelial cells resist lymphokine-activated killer cell-mediated injury. J. Immunol. 143(7): 2407, 1989. 